\title{
Associação entre prática religiosa e estilo de vida saudável em escolares de Pelotas, RS
}

\author{
Association between religious practice and healthy lifestyle in students from \\ Pelotas, RS, Southern Brazil
}

Léo Dutra Cabistany ${ }^{1}$, Airton José Rombaldi ${ }^{1,2}$

${ }^{1}$ Programa de Pós-graduação em Educação Física da Universidade Federal de Pelotas (UFPel) - Pelotas (RS), Brasil.

${ }^{2}$ Grupo de Estudos em Epidemiologia da Atividade Física (GEEAF) da UFPel - Pelotas (RS), Brasil.

DOI: http://dx.doi.org/10.7322/abcshs.v39i2.624

\begin{abstract}
RESUMO
Introdução: Dentre as condutas que podem influenciar positivamente a adoção e sustentação de hábitos saudáveis está a prática religiosa ou influência de uma religião, em função da adoção de princípios éticos. Objetivo: O estudo objetivou associar prática religiosa e estilo de vida saudável em adolescentes de terceiro ano do ensino médio de duas escolas públicas de Pelotas, Rio Grande do Sul, Brasil. Métodos: Trata-se de um estudo observacional de caráter transversal, com amostra de 183 estudantes, com idade mínima de 15 anos, de ambos os sexos, matriculados em duas escolas públicas estaduais da cidade de Pelotas, selecionados por conveniência. Os escolares responderam a instrumento autoaplicado contendo 118 questões e os desfechos foram relacionados à prática religiosa. Resultados: Houve associação limítrofe entre a variável idade que teve a primeira relação sexual e frequência ao culto religioso $(p=0,05)$. Além disso, o estudo mostrou associações significantes entre ter recebido instrução religiosa e ingestão de álcool nos últimos 30 dias $(p=0,03)$; e entre tempo assistindo televisão maior que duas horas e tempo de prática religiosa maior de 10 anos $(p=0,04)$. Conclusão: $O$ estudo encontrou associações distintas entre a prática religiosa e suas variáveis dependentes. Essa área de estudos deve ser investigada de forma mais abrangente, em amostras maiores e representativas, para que se possa ter evidências mais objetivas a respeito da prática religiosa e sua potencial influência sobre a saúde física dos indivíduos que professam cada tipo de crença religiosa.
\end{abstract}

Palavras-chave: religião; fatores de risco; estilo de vida; adolescente.

\begin{abstract}
Introduction: Among the approaches that can positively influence the adoption and maintenance of healthy habits, the religious practice or the influence of religion seems to be important due to the adoption of ethical principles. Objective: This study aimed at evaluating the potential association between religious practice and healthy lifestyle among adolescents attending the last year of high school from two public schools in Pelotas, Rio Grande do Sul, Brazil. Methods: Cross-sectional study with a convenience sample of 183 students, aged at least 15 years old, including boys and girls. The students answered a selfadministered instrument containing 118 questions; the outcomes were related to religious practice. Result: There was a borderline association between the age of sexual initiation and frequency to religious cults $(p=0.05)$. Moreover, the study showed significant associations between having received religious instruction and drinking alcohol in the last 30 days $(p=0.03)$; and between watching TV for more than 2 hours and being engaged in religious practice for more than 10 years $(p=0.04)$. Conclusion: The study found different associations between religious practice and its dependent variables. This area of study should be more thoroughly investigated in larger, representative samples, so that we can provide more objective evidence about religious practice and its potential influence on the physical health of individuals who profess every sort of religious belief.
\end{abstract}

Keywords: religion; risk factors; life style; adolescent.

Recebido em: 13/05/2013

Revisado em: 19/12/2013

Aprovado em: 28/05/2014

Endereço para correspondência

Airton José Rombaldi - Rua Luis de Camões, 625 - Areal - CEP: 96055-630 - Pelotas (RS), Brasil - E-mail: rombaldi@ufpel.tche.br

Conflito de interesses: nada a declarar. 


\section{INTRODUÇÃO}

Doenças com causas evitáveis são as principais causadoras de morte e adoecimento. Essas doenças crônicas não transmissíveis (DANT's), no ano de 2005, foram responsáveis por 35 milhões de óbitos, cerca de $60 \%$ do número de mortes ao redor do mundo. Estima-se que até o ano de 2015 haja um acréscimo de 17\% no total de mortes em decorrência de doenças causadas por hábitos não saudáveis ${ }^{1}$.

Hábitos saudáveis, como a prática de regular de atividade física (AF), alimentação adequada, abstenção de drogas e prática sexual segura, são indispensáveis a todos ${ }^{2}$ e esses cuidados devem ser estimulados desde a infância e adolescência ${ }^{3}$, de modo a prevenir o aparecimento de DANT's. Além disso, há a necessidade de expandir o conhecimento de determinados comportamentos que se destacam como principal causa de morte e adoecimento ${ }^{4}$, assim como também todo o tipo de fenômeno que interfere e/ou se relaciona com atitudes nocivas à saúde.

Dentre as condutas que podem influenciar positivamente a adoção e sustentação de hábitos saudáveis está a prática religiosa ou influência de uma religião, em função da adoção de princípios éticos. Nesse sentido, estudo conduzido por Stroppa e Almeida ${ }^{5}$ demonstrou que o envolvimento religioso influencia a saúde em diversos aspectos, oferecendo suporte social, além de direção e orientação espiritual.

Anjos e Saneto ${ }^{6}$ realizaram estudo com líderes de cinco congregações cristãs, de modo a saber suas opiniões e também das igrejas as quais representavam sobre: como encaravam a AF como fator promotor de saúde, se ofereciam AF para os membros e se havia alguma oposição por parte da igreja quanto a alguma prática. Os líderes se mostraram favoráveis à prática de AF, por vezes promovendo tais práticas. Os autores concluíram que havia apoio das lideranças religiosas para adoção de estilo de vida ativo, apesar de não apoiarem todas as práticas indiscriminadamente ${ }^{6}$.

Alguns estudos realizados mostraram associações positivas entre prática religiosa e proteção contra doenças mentais ${ }^{7-9}$. Porém, poucos associaram prática de uma religião e prevenção para doenças físicas, incluindo as doenças crônicas. Um desses foi realizado com 3.056 habitantes de Israel, através de inquérito telefônico. Como principal achado, os autores reportaram que diversos comportamentos de risco foram minimizados e/ou desapareceram por influência da prática religiosa ${ }^{10}$. Há também estudos brasileiros relatando que a prática religiosa reduziu o consumo de drogas lícitas e ilícitas ${ }^{11}$ e o consumo de alimentos não saudáveis ${ }^{12}$, reforçando a hipótese de que a religião aparece como fator protetor contra os fatores de risco que levam às doenças crônicas.

As religiões podem exercer influência no desenvolvimento de atitudes, crenças e valores das pessoas, mesmo entre as mais jovens ${ }^{5}$, ainda assim, há escassez de estudos no Brasil em relação à associação de comportamentos saudáveis e crença religiosa, de modo que não se pode compreender de forma clara a relação entre essas variáveis. Assim sendo, este estudo objetivou analisar a influência da prática religiosa sobre comportamentos saudáveis em escolares do município de Pelotas, Rio Grande do Sul.

\section{MÉTODOS}

Estudo transversal desenvolvido com escolares do terceiro ano do ensino médio, com idade mínima de 15 anos, de ambos os sexos, matriculados em duas escolas públicas estaduais da cidade de Pelotas, Rio Grande do Sul, selecionados por conveniência. Tais escolas foram selecionadas por serem instituições de referência educacional na cidade, em função de atenderem alunos de todos os níveis socioeconômicos e de ensino (da educação infantil até o terceiro ano do ensino médio).

Inicialmente, foi realizado contato com a $5^{\mathrm{a}}$ Coordenadoria Regional de Educação ( $5^{\mathrm{a}} \mathrm{CRE}$ ), para que pudessem compreender o estudo a ser realizado, bem como autorizar a coleta de dados nas escolas. Posteriormente, foi entregue à direção das escolas uma cópia da autorização da $5^{\mathrm{a}} \mathrm{CRE}$ e em seguida foi estabelecido contato com os escolares nos quais o instrumento foi aplicado, a fim de explicar os procedimentos e esclarecer dúvidas a respeito da pesquisa, bem como apresentar e entregar o Termo de Consentimento Livre e Esclarecido, que foi assinado pelo responsável do aluno.

Como critérios de inclusão para participação no estudo, o aluno precisava estar devidamente matriculado em uma das turmas de terceiro ano do ensino médio das escolas selecionadas e trazer o Termo de Consentimento Livre e Esclarecido assinado por pais ou responsáveis.

O instrumento autoaplicado contendo 118 questões foi utilizado na pesquisa para verificar variáveis demográficas, socioeconômicas, nutricionais e comportamentais. As variáveis demográficas foram: idade (coletada em anos completos), sexo e cor da pele (branca e não branca); as socioeconômicas foram: escolaridade (coletada em anos completos de estudo) e nível socioeconômico (medido através do instrumento da Associação Brasileira de Empresas de Pesquisas, ABEP) ${ }^{13}$. Esse instrumento classificou os respondentes em classes econômicas de A (mais elevada) até E, de acordo com bens de consumo existentes no domicílio, escolaridade do chefe da família e presença de empregada doméstica mensalista.

$\mathrm{O}$ estado nutricional foi determinado pelo índice de massa corporal (IMC) dos escolares, calculado a partir do peso e altura autorreferidos, e categorizado em eutrófico, sobrepeso e obesidade ${ }^{14,15}$. O nível de AF foi mensurado através do instrumento de Bastos et al..$^{16}$ e o ponto de corte utilizado para considerar os adolescentes ativos foi de $300 \mathrm{~min}^{17} \mathrm{de} \mathrm{AF}$ semanais. Indivíduos que relataram zero minuto de AF semanal no lazer e deslocamento foram considerados sedentários. Valores superiores a zero e menores do que $300 \mathrm{~min}$ foram classificados como insuficientemente ativos ${ }^{17}$.

A atividade sedentária foi medida através das perguntas: "Em um dia que você vai para a escola, quantas horas de TV você assiste?" 
(respostas categorizadas em $0-2$ e 3 ou mais horas por dia); "Em um dia que você vai para a escola, quantas horas você joga video game ou usa o computador para alguma atividade que não seja trabalho escolar?" (foram incluídas atividades como Playstation, games no computador e Internet e as respostas foram categorizadas em 0-2 e 3 ou mais horas por dia).

Consumo de tabaco e álcool foi medido através da pergunta "Consumiu pelo menos uma vez por dia nos últimos 30 dias?" (sendo as respostas categorizadas em sim e não). O comportamento sexual de risco foi medido através da pergunta "Idade em que teve a primeira relação sexual?" ${ }^{\text {. }}$.

Para avaliar o conhecimento dos indivíduos, foi utilizado um questionário elaborado por Borges et al. ${ }^{19}$. O instrumento objetiva avaliar o conhecimento populacional sobre a influência de quatro fatores de risco para DANT (sedentarismo, tabagismo, consumo excessivo de álcool e alimentação inadequada) e oito morbidades (diabetes, hipertensão arterial, AIDS, osteoporose, câncer de pulmão, depressão, cirrose hepática e infarto agudo do miocárdio). A definição de certo ou errado para cada resposta foi baseada em revisão da literatura sobre a associação de cada fator de risco e doença ou agravo, com preferência para revisões sistemáticas e metanálises ${ }^{19}$. Para cada um dos quatro fatores, foi construído um escore de acertos, que poderia variar de zero a oito pontos. O escore final de conhecimento da relação de cada fator de risco com as DANT foi dicotomizado (escores entre 0 e 6 pontos foram considerados como não tendo conhecimento e escores entre $7 \mathrm{e}$ 8 pontos foram considerados como tendo conhecimento).

Os desfechos deste estudo foram relacionados à prática religiosa. O instrumento foi criado pelos autores com base em literatura já existente, sendo utilizadas as seguintes questões para determiná-los: (1) O entrevistado possui religião? (sim/não); (2) Em caso de resposta positiva na primeira pergunta, qual religião o entrevistado professa?; (3) Em caso de resposta positiva na primeira pergunta, o respondente é praticante "regular"? (foi considerado praticante regular o entrevistado que relatou frequência ao menos semanal ao evento religioso); (4) Em caso de resposta positiva na primeira pergunta, há quanto tempo o respondente frequenta o culto religioso?; (5) Recebeu instrução religiosa na infância? (sim/não).

Foi realizado estudo piloto em uma turma de terceiro ano do ensino médio de outra escola da rede pública estadual de ensino da cidade de Pelotas, Rio Grande do Sul, a qual não integrou a amostra, de modo a estimar o tempo necessário para responder ao instrumento, bem como a aplicabilidade do estudo. A coleta de dados ocorreu em sala de aula e foi realizada pelos pesquisadores. Após o preenchimento do questionário, os alunos os colocaram em envelopes, que foram lacrados.

Os pais e/ou responsáveis dos escolares, ou os próprios, no caso de serem maiores de 18 anos, receberam um Termo de Consentimento Livre e Esclarecido, que lhes possibilitou decidir participar ou não do mesmo. Este estudo foi submetido ao Comitê de Ética da Escola Superior de Educação Física da Universidade
Federal de Pelotas (UFPel) e foi aprovada a realização do projeto sob protocolo $\mathrm{n}^{\circ}$ 019/2011.

Os escores foram digitados numa planilha Excel e, após a checagem para a ocorrência de erros, foram transferidos para o software estatístico STATA 10.0. Foi utilizada a estatística descritiva (para cálculo de proporções) e inferencial (testes do $\chi^{2}$ e exato de Fisher). O nível de significância aceito foi de $\mathrm{p}<0,05$.

\section{RESULTADOS}

A Tabela 1 mostra as variáveis sociodemográficas da amostra. O número total de participantes no presente estudo foi de 159 respondentes $(18,8 \pm 5,1$ anos), sendo a maioria do sexo feminino $(66,0 \%)$. Quanto à variável nível socioeconômico, a maioria dos estudantes foi classificada no nível B $(52,4 \%)$ e, em relação à variável "com quem reside", a categoria mais prevalente indicou que os respondentes moravam com pai e mãe (50,4\%). Com relação à cor da pele, a maioria se descreveu como tendo cor de pele branca $(76,3 \%)$; ensino médio completo $(37,2 \%)$ foi a categoria

Tabela 1: Descrição sociodemográfica de estudantes de ensino médio de duas grandes escolas públicas de Pelotas, Rio Grande do Sul, Brasil $(n=159)$

\begin{tabular}{|c|c|c|}
\hline \multirow{2}{*}{ Variáveis } & \multicolumn{2}{|c|}{ Total } \\
\hline & $\mathbf{n}$ & $\%$ \\
\hline \multicolumn{3}{|l|}{ Sexo $(n=159)$} \\
\hline Masculino & 54 & 33,9 \\
\hline Feminino & 105 & 66,1 \\
\hline \multicolumn{3}{|l|}{ Idade (anos) ( $\mathrm{n}=159)$} \\
\hline $15-16$ & 37 & 23,3 \\
\hline $17-18$ & 101 & 63,5 \\
\hline $19-20$ & 21 & 13,2 \\
\hline \multicolumn{3}{|l|}{ Nível socioeconômico ( $n=124)$} \\
\hline A & 4 & 3,2 \\
\hline B & 65 & 52,4 \\
\hline C & 55 & 44,4 \\
\hline \multicolumn{3}{|l|}{ Com quem reside $(n=139)$} \\
\hline Pai somente & 6 & 4,3 \\
\hline Mãe somente & 44 & 31,7 \\
\hline Ambos & 70 & 50,4 \\
\hline Outros & 19 & 13,6 \\
\hline \multicolumn{3}{|l|}{ Cor da pele $(n=135)$} \\
\hline Branca & 103 & 76,3 \\
\hline Não branca & 32 & 25,7 \\
\hline \multicolumn{3}{|l|}{ Escolaridade chefe da família $(n=129)$} \\
\hline Analfabeto/fundamental incompleto & 43 & 33,3 \\
\hline Ensino fundamental completo & 26 & 20,2 \\
\hline Ensino médio completo & 48 & 37,2 \\
\hline Ensino superior completo & 12 & 9,3 \\
\hline \multicolumn{3}{|l|}{ Quem é o chefe da família ( $n=139)$} \\
\hline Pai/padrasto & 69 & 49,7 \\
\hline Mãe & 48 & 34,6 \\
\hline Avós & 7 & 5,0 \\
\hline Próprio aluno & 11 & 7,9 \\
\hline Outro & 4 & 2,8 \\
\hline
\end{tabular}


mais observada para a variável "escolaridade do chefe da família". Quanto ao "chefe da família", a categoria de respostas mais relatada foi "o pai/padrasto" (49,6\%).

A Tabela 2 apresenta a prevalência de variáveis de risco à saúde de acordo com instrução religiosa recebida na infância, em escolares de Pelotas, Rio Grande do Sul. A única variável que apresentou significância estatística $(\mathrm{p}=0,03)$ com prática religiosa foi "a ingestão de pelo menos uma dose de álcool nos últimos 30 dias".

A Tabela 3 apresenta a prevalência de variáveis de risco para doenças crônicas segundo a frequência ao culto religioso em escolares de Pelotas, Rio Grande do Sul. Houve significância estatística apenas limítrofe $(\mathrm{p}=0,05)$ na associação entre a variável independente "idade que teve a primeira relação sexual" e frequência ao culto.

A Tabela 4 apresenta as variáveis de risco para doenças crônicas de acordo com o tempo em anos de prática religiosa em escolares de Pelotas, Rio Grande do Sul. Houve resultado estatisticamente significativo $(\mathrm{p}<0,04)$ para a variável "tempo assistindo televisão nos dias em que tem aula". O resultado mostrou que o tempo assistindo televisão foi maior para os escolares que possuem religião há 10 anos ou mais.

\section{DISCUSSÃO}

Os dados do presente estudo se mostraram diferentes, em alguns resultados, da maioria dos estudos que associaram prática religiosa e estilo de vida saudável. Neste trabalho, os entrevistados que receberam instrução religiosa na infância apresentaram maior consumo de álcool. Esse resultado não tem fácil explicação, mas, provavelmente, os cultos religiosos não incentivaram o consumo de álcool, mas ao não incentivarem a abstinência, podem ter contribuído para o comportamento. Além de haver a possibilidade de o resultado ter sido encontrado ao acaso, visto se tratar de estudo transversal. Nossos resultados vem de encontro aos estudos de Sanchez et al. ${ }^{20}$, que mostraram a prática religiosa como fator protetor nas respostas de $75 \%$ dos jovens entrevistados; e de Chen et al. ${ }^{21}$, os quais relataram que maiores níveis de prática religiosa, em estudantes, foram inversamente associados aos primeiros estágios de experimentação de drogas e menos oportunidade de exposição ao álcool.

Jovens com alta devoção religiosa tendem a evitar comportamentos sexuais de risco e a iniciação sexual precoce. Estudo de Cerqueira-Santos ${ }^{22}$ mostrou que, à medida que aumentava o grau de devoção religiosa de adolescentes, mais tardia foi sua iniciação sexual. Esse achado confirma os resultados do presente estudo,

Tabela 2: Prevalência de variáveis de risco à saúde de acordo com instrução religiosa recebida na infância, em escolares de Pelotas, Rio Grande do Sul, Brasil

\begin{tabular}{|c|c|c|c|c|c|}
\hline & \multicolumn{4}{|c|}{ Recebeu instrução religiosa } & \multirow{3}{*}{ Valor $\mathrm{F}$} \\
\hline & \multicolumn{2}{|c|}{ Sim } & \multicolumn{2}{|c|}{ Não } & \\
\hline & $\mathbf{n}$ & $\%$ & $\mathbf{n}$ & $\%$ & \\
\hline Prática de atividade física no lazer & & & & & $0,7^{\star}$ \\
\hline Não & 56 & 66,7 & 19 & 70,4 & \\
\hline Sim & 28 & 33,3 & 8 & 29,6 & \\
\hline $\mathrm{IMC}\left(\mathrm{kg} / \mathrm{m}^{2}\right)$ & & & & & $0,4^{*}$ \\
\hline Normal & 75 & 76,5 & 21 & 65,6 & \\
\hline Sobrepeso & 19 & 19,4 & 9 & 28,1 & \\
\hline Obesidade & 4 & 4,1 & 2 & 6,3 & \\
\hline Fumou pelo menos um cigarro nos últimos 30 dias & & & & & $0,7^{\#}$ \\
\hline Não & 105 & 93,8 & 37 & 92,5 & \\
\hline Sim & 7 & 6,2 & 3 & 7,5 & \\
\hline Ingeriu pelo menos uma dose de álcool nos últimos 30 dias & & & & & $0,03^{*}$ \\
\hline Não & 41 & 35,6 & 21 & 55,3 & \\
\hline Sim & 74 & 64,4 & 17 & 44,7 & \\
\hline Idade que teve a primeira relação sexual & & & & & $0,5^{\star}$ \\
\hline Até os 15 anos & 94 & 75,3 & 31 & 24,8 & \\
\hline Após os 15 anos & 12 & 85,7 & 2 & 14,2 & \\
\hline Tempo assistindo televisão nos dias em que tem aula & & & & & $0,6^{*}$ \\
\hline Até 2 horas/dia & 50 & 42,7 & 15 & 38,5 & \\
\hline Mais de 2 horas/dia & 67 & 57,3 & 24 & 61,5 & \\
\hline $\begin{array}{l}\text { Tempo usando computador/ jogando video game } \\
\text { nos dias em que tem aula }\end{array}$ & & & & & $0,2^{*}$ \\
\hline Até 2 horas/dia & 19 & 46,3 & 19 & 33,9 & \\
\hline Mais de 2 horas/dia & 22 & 53,7 & 37 & 66,1 & \\
\hline
\end{tabular}

* Teste do $\chi^{2}$; IMC: índice de massa corporal; \# Teste exato de Fisher 
Tabela 3: Prevalência de variáveis de risco para doenças crônicas segundo frequência ao culto religioso em escolares de Pelotas, Rio Grande do Sul, Brasil

\begin{tabular}{|c|c|c|c|c|c|}
\hline & \multicolumn{4}{|c|}{ Frequência ao culto Religioso } & \multirow{3}{*}{ Valor $p$} \\
\hline & \multicolumn{2}{|c|}{ Diária/semanal } & \multicolumn{2}{|c|}{ Eventualmente } & \\
\hline & $\mathbf{n}$ & $\%$ & $\mathbf{n}$ & $\%$ & \\
\hline Prática de atividade física no lazer & & & & & $0,6^{*}$ \\
\hline Não & 23 & 71,9 & 31 & 67,4 & \\
\hline Sim & 9 & 28,1 & 15 & 32,6 & \\
\hline IMC $\left(\mathrm{kg} / \mathrm{m}^{2}\right)$ & & & & & $0,5^{*}$ \\
\hline Normal & 27 & 75,0 & 45 & 83,3 & \\
\hline Sobrepeso & 7 & 19,4 & 8 & 14,8 & \\
\hline Obesidade & 2 & 5,6 & 1 & 1,9 & \\
\hline Fumou pelo menos um cigarro nos últimos 30 dias & & & & & $0,4^{\#}$ \\
\hline Não & 38 & 97,4 & 52 & 94,5 & \\
\hline Sim & 1 & 2,6 & 3 & 5,5 & \\
\hline Ingeriu pelo menos uma dose de álcool nos últimos 30 dias & & & & & $0,6^{*}$ \\
\hline Não & 17 & 42,5 & 22 & 37,9 & \\
\hline Sim & 23 & 57,5 & 36 & 62,1 & \\
\hline Idade que teve a primeira relação sexual & & & & & $0,05^{\#}$ \\
\hline Até os 15 anos & 33 & 41,9 & 54 & 67,7 & \\
\hline Após os 15 anos & 8 & 58,1 & 4 & 33,3 & \\
\hline Tempo assistindo televisão nos dias em que tem aula & & & & & $0,1^{*}$ \\
\hline Até 2 horas/dia & 18 & 43,9 & 18 & 31,0 & \\
\hline Mais de 2 horas/dia & 23 & 56,1 & 40 & 69,0 & \\
\hline Tempo usando computador/ jogando video game nos dias em que tem aula & & & & & $0,2^{\#}$ \\
\hline Até 2 horas/dia & 19 & 46,4 & 19 & 33,9 & \\
\hline Mais de 2 horas/dia & 22 & 53,6 & 37 & 66,1 & \\
\hline
\end{tabular}

*Teste do $\chi^{2}$; "teste exato de Fisher; IMC: índice de massa corporal

Tabela 4: Prevalência de variáveis de risco para doenças crônicas segundo tempo em anos de prática religiosa, em escolares de Pelotas, Rio Grande do Sul, Brasil

\begin{tabular}{|c|c|c|c|c|c|}
\hline & \multicolumn{4}{|c|}{ Tempo em anos de prática religiosa } & \multirow{3}{*}{ Valor $p$} \\
\hline & \multicolumn{2}{|c|}{ Até 10 anos } & \multicolumn{2}{|c|}{ Mais de 10anos } & \\
\hline & n & $\%$ & $\mathbf{n}$ & $\%$ & \\
\hline Prática de atividade física no lazer & & & & & $0,6^{*}$ \\
\hline Não & 13 & 61,9 & 20 & 69,0 & \\
\hline Sim & 8 & 38,1 & 9 & 31,0 & \\
\hline $\mathrm{IMC}\left(\mathrm{kg} / \mathrm{m}^{2}\right)$ & & & & & $0,4^{\#}$ \\
\hline Normal & 18 & 58,1 & 27 & 84,4 & \\
\hline Sobrepeso & 11 & 35,5 & 4 & 12,5 & \\
\hline Obesidade & 2 & 6,4 & 1 & 3,1 & \\
\hline Fumou pelo menos um cigarro nos últimos 30 dias & & & & & $0,3^{\#}$ \\
\hline Não & 30 & 90,9 & 34 & 97,1 & \\
\hline Sim & 3 & 9,1 & 1 & 2,9 & \\
\hline Ingeriu pelo menos uma dose de álcool nos últimos 30 dias & & & & & $0,9^{*}$ \\
\hline Não & 12 & 35,3 & 12 & 34,3 & \\
\hline Sim & 22 & 64,7 & 23 & 64,7 & \\
\hline Idade que teve a primeira relação sexual & & & & & $0,8^{*}$ \\
\hline Até os 15 anos & 10 & 47,6 & 12 & 50,0 & \\
\hline Após os 15 anos & 11 & 52,4 & 12 & 50,0 & \\
\hline Tempo assistindo televisão nos dias em que tem aula & & & & & $0,04^{*}$ \\
\hline Até 2 horas/dia & 18 & 52,9 & 11 & 29,7 & \\
\hline Mais de 2 horas/dia & 16 & 47,1 & 26 & 70,3 & \\
\hline Tempo usando computador/ jogando vídeo game nos dias em que tem aula & & & & & $0,7^{*}$ \\
\hline Até 2 horas/dia & 15 & 44,1 & 17 & 47,2 & \\
\hline Mais de 2 horas/dia & 19 & 55,9 & 19 & 52,8 & \\
\hline
\end{tabular}

*Teste do $\chi^{2}$; " teste exato de Fisher; IMC: índice de massa corporal. 
pois mostra que os escolares com frequência diária/semanal ao culto têm iniciação sexual em período posterior.

No presente estudo, atividades sedentárias como "tempo assistindo televisão nos dias em que tem aula" mostrou associação limítrofe $(\mathrm{p}=0,05)$ com frequência ao culto religioso. Estudo conduzido por Sarriera et al..$^{23}$ mostrou que, nos dias em que vão à escola, os jovens gastam em torno de $10 \%$ do dia (cerca de 2 horas e 15 minutos) assistindo televisão, representando assim, risco para saúde cardiovascular. Ainda, os entrevistados que apresentaram mais de dez anos de devoção religiosa foram os que relataram maior tempo gasto assistindo televisão, assemelhando-se aos dados do estudo citado. De forma semelhante, estudo conduzido em Pernambuco mostrou que jovens que se diziam religiosos tendiam a ser insuficientemente ativos, além de apresentar comportamento sedentário nos finais de semana ${ }^{24}$. Identifica-se também tendência de que indivíduos que professam religião evangélica apresentem chance maior de não participar das aulas de educação física ${ }^{24}$.

A amostra estudada apresentou dois e três fatores de risco com prevalências elevadas. Possivelmente, pode-se associar essa elevada prevalência com duas variáveis do presente estudo que mostraram significância estatística e tem a prática religiosa como fator de exposição. Essas variáveis foram consumo abusivo de álcool e tempo assistindo televisão. Os resultados sugerem que os cultos religiosos na cidade de Pelotas possivelmente não dão atenção suficiente a esses comportamentos.

A formação religiosa, por ser algo também bastante amplo e individual, além de um fenômeno cultural e histórico, pode não ter sido abordada de forma completa, pois há um número elevado de religiões, cada uma com seus costumes, práticas e maneiras de se comunicar com seus membros. O presente estudo sugere por seus resultados que, possivelmente, as religiões na cidade de Pelotas não priorizam a saúde física de seus fiéis ou apenas alguns comportamentos são tidos como importantes em detrimento de outros. Nesse sentido, os resultados do presente estudo divergem do relato de Silva et al. ${ }^{25}$, os quais relataram que indivíduos religiosos do estado de São Paulo tendem a apresentar menos fatores de risco para doenças crônicas e melhores condições de saúde do que a população em geral.

A literatura tem mostrado associações significantes entre prática religiosa e comportamentos preventivos em saúde ${ }^{26}$. No entanto, considerável parcela das pesquisas foi realizada com metodologia transversal, dificultando a compreensão de qual variável ocorreu primeiro. Assim, estudos mais elaborados são necessários para compreender adequadamente as relações de causa e efeito ${ }^{26}$.

Algumas limitações precisam ser apresentadas. O estudo transversal determina a coleta dos desfechos e das exposições ao mesmo tempo, o que eventualmente pode incorrer em vieses. Além disso, a amostra de conveniência e a falta de cálculo amostral reduziram a possibilidade de generalização dos resultados.

Conclui-se que o estudo encontrou associações distintas entre a prática religiosa e suas variáveis dependentes. $\mathrm{O}$ tema se mostrou como fator protetor para alguns comportamentos de risco entre os escolares estudados, mas por vezes foi associado a condutas de risco para a saúde.

Essa área de estudo deve ser investigada de forma mais abrangente, em amostras maiores e representativas, para que se possa ter evidências mais objetivas a respeito da prática religiosa e sua potencial influência sobre a saúde física dos indivíduos que professam cada tipo de crença religiosa.

\section{REFERÊNCIAS}

1. World Health Organization (WHO). Interventions on diet and physical activity: what works. Summary Report. Geneve: WHO; 2009

2. Guedes DP, Grondin LMV. Percepção de hábitos saudáveis por adolescentes: associação com indicadores alimentares, prática de atividade física e controle de peso corporal. Rev Bras Ciên Esp. 2002;24(1):23-45.

3. Farias Júnior JC, Nahas MV, Barros MVG, Loch MR, Oliveira ESA, De Bem MFL, et al. Comportamentos de risco à saúde em adolescentes no Sul do Brasil: prevalência e fatores associados. Rev Panam Salud Publica. 2009;25(4):344-52. http://dx.doi.org/10.1590/S1020-49892009000400009

4. Carlini-Cotrim B, Gazal-Carvalho C, Gouveia N. Comportamentos de saúde entre jovens estudantes das redes pública e privada da área metropolitana do Estado de São Paulo. Rev Saúde Pública. 2000;34(6):636-45.

http://dx.doi.org/10.1590/S0034-89102000000600012

5. Stroppa A, Almeida AM. Saúde e espiritualidade: uma nova visão da medicina. In: Salgado MI, Freire G. (Orgs.). Saúde e espiritualidade: uma nova visão da medicina. Belo Horizonte: Inede; 2008. p. 427-43.
6. Anjos JL, Saneto JG. Práticas corporais e religiosidade: discursos de líderes religiosos. Coleç Pesqui Educ Fís. 2007;6(2):171-8.

7. Dalgalarrondo P. Estudos sobre religião e saúde mental realizados no Brasil: histórico e perspectivas atuais. Rev Psiq Clín. 2007;34(1):25-33.

http://dx.doi.org/10.1590/S0101-60832007000700005

8. Gastaud MB, Souza LDM, Braga L, Horta CL, Oliveira FM, Sousa $P L R$, et al. Bem-estar espiritual e transtornos psiquiátricos menores em estudantes de psicologia: estudo transversal. Rev Psiquiatr Rio Gd Sul. 2006; 28(1):12-8.

http://dx.doi.org/10.1590/S0101-81082006000100003

9. Moraes PAC, Dalgalarrondo P. Mulheres encarceradas em São Paulo: saúde mental e religiosidade. J Bras Psiquiatr. 2006; 55(1):50-6. http://dx.doi.org/10.1590/S0047-20852006000100007

10. Shmueli A, Tamir D. Health behavior and religiosity among Israeli jews. Isr Med Assoc J. 2007;9(10):703-7.

11. Dalgalarrondo $P$, Soldera MA, Corrêa Filho HR, Silva CAM Religião e uso de drogas por adolescentes. Rev Bras Psiquiatr. 2004:26(2):82-90.

http://dx.doi.org/10.1590/S1516-44462004000200004 
12. Rodrigues EM, Boog MCF. Problematização como estratégia de educação nutricional com adolescentes obesos. Cad Saúde Pública. 2006;22(5)923-31. http://dx.doi.org/10.1590/S0102-311X2006000500005

13. Associação Brasileira de Empresas de Pesquisa. Critério de classificação econômica do Brasil 2014. Disponível em: http:// www.abep.org. Acesso em: 02/07/2014. Acesso em: 10 out 2011.

14. World Health Organization (WHO). Physical Status: the use and interpretation of anthropometry. WHO Technical Report Series $n^{\circ}$ 854. Geneva: WHO; 1995.

15. Cole TJ, Bellizzi MC, Flegal KM, Dietz WH. Establishing a standard definition for child overweight and obesity worldwide: international survey. BMJ. 2000;320(7244):1240-3

http://dx.doi.org/10.1136/bmj.320.7244.1240

16. Bastos JP, Araújo CL, Hallal PC. Prevalence of insufficient physical activity and associated factors in Brazilian adolescents. J Phys Act Health. 2008;5(6):777-94.

17. U.S. Department of Health and Human Service. 2008 Physical Activity Guidelines for Americans. Disponível em: http://www.health. gov/paguidelines/pdf/paguide.pdf. Acesso em: 01 jun. 2011.

18. Eaton DK, Kann L, Kinchen S, Shanklin S, Ross J, Hawkins J, et al. Youth risk behavior surveillance: United States, 2009. MMWR Surveill Summ. 2010; 59(SS05):1-142.

19. Borges TT, Rombaldi AJ, Knuth AG, Hallal PC. Conhecimento sobre fatores de risco para doenças crônicas: estudo de base populacional. Cad Saúde Pública. 2009;25(7):1511-20. http://dx.doi.org/10.1590/S0102-311X2009000700009
20. Sanchez ZVM, Oliveira LG, Nappo SA. Fatores protetores de adolescentes contra o uso de drogas com ênfase na religiosidade. Ciên Saúde Col. 2004; 9(1):43-55.

http://dx.doi.org/10.1590/S1413-81232004000100005

21. Chen CY, Dormitzer CM, Bejarano J, Anthony JC. Religiosity and the earliest stages of adolescents drug involvement in seven countries of Latin America. Am J Epidemiol. 2004;159(12):1180-8. http://dx.doi.org/10.1093/aje/kwh151

22. Cerqueira-Santos E. Comportamento sexual e religiosidade: um estudo com jovens Brasileiros. Tese (Doutorado) - Instituto de Psicologia da Universidade Federal do Rio Grande do Sul, Porto Alegre, 2008. Disponível em: http://hdl.handle. net/10183/13113?locale=pt_BR Acesso em: 27 out. 2011.

23. Sarriera JC, Tatim DC, Coelho RPS, Bücker J. Uso do tempo livre por adolescentes de classe popular. Psicol Reflex Crit. 2007;20(3):361-7. http://dx.doi.org/10.1590/S0102-79722007000300003

24. Mélo EN, Meneses AS, Silva Júnior AG, Wanderley Júnior RS Barros MVG. Associação entre religiosidade, atividade física e comportamento sedentário em adolescentes. Rev Bras Ativ Fis Saúde. 2013;17(5):359-69. http://dx.doi.org/10.12820/2317-1634.2012v17n5p359

25. Silva LBE, Silva SSBE, Marcílio AG, Pierin AMG. Prevalência de hipertensão arterial em Adventistas do Sétimo Dia da capital e do interior paulista. Arq Bras Cardiol. 2012;98(4):329-37. http://dx.doi.org/10.1590/S0066-782X2012005000018

26. Vasconcelos EM. A associação entre vida religiosa e saúde: uma breve revisão de estudos quantitativos. RECIIS. 2010;4(3):12-8. http://dx.doi.org/10.3395/reciis.v4i3.381pt 